\title{
Functional posterior communicating artery of patients with posterior circulation ischemia using phase contrast magnetic resonance angiography
}

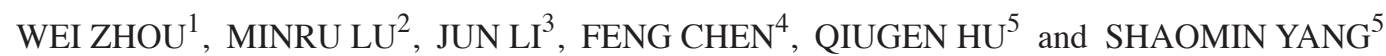 \\ Departments of ${ }^{1}$ Nuclear Medicine and ${ }^{2}$ Rehabilitation Medicine, ${ }^{3}$ VIP Medical Center, ${ }^{4}$ Medical Record Statistics Office \\ and ${ }^{5}$ Department of Radiology, Shunde Hospital, Southern Medical University (The First People's Hospital of \\ Shunde Foshan), Foshan, Guangdong 528308, P.R. China
}

Received April 13,2018; Accepted October 4, 2018

DOI: $10.3892 /$ etm.2018.6897

\begin{abstract}
Posterior communicating artery (PCoA) in patients with posterior circulation ischemia (PCI) was quantitatively studied using phase contrast magnetic resonance angiography (PC-MRA). Fifty-one cases who received PC-MRA were included in the study, and divided into the research and control groups. The mean flow volume, mean flow velocity, minimum flow volume, maximum flow volume, minimum flow velocity and maximum flow velocity of the basal artery (BA), bilateral vertebral arteries, internal carotid arteries and functional posterior communicating artery (F-PCoA) were recorded, the peak heights of flow volume and flow velocity were calculated, and the typing of F-PCoA was analyzed, followed by statistical analysis. Fifty-two F-PCoAs were detected, and the median values of mean flow volume, mean flow velocity, cross-sectional area and lumen diameter were $20.31 \mathrm{ml} / \mathrm{min}$, $4.01 \mathrm{~cm} / \mathrm{sec}, 0.08 \mathrm{~cm}^{2}$ and $0.16 \mathrm{~cm}$, respectively. The blood flow curve of F-PCoA showed the sawtooth-like changes, and there could be either unidirectional blood flow or bidirectional blood flow in one cardiac cycle. F-PCoA was divided into the following 3 types: F-PCoA was consistent with anatomical PCoA (A-PCoA), F-PCoA was inconsistent with A-PCoA, and mixed type. In the presence of F-PCoA, both the diameter and cross-sectional area of BA were small, and the maximum flow velocity and peak height of flow volume were reduced, but there was no necessary correlation with the occurrence of PCI. Both flow volume and flow velocity of BA in the research group were reduced, and the forward posterior shunt flow of F-PCoA was increased. Hemodynamic characteristics of
\end{abstract}

Correspondence to: Dr Wei Zhou, Department of Nuclear Medicine, Shunde Hospital, Southern Medical University (The First People's Hospital of Shunde Foshan), 1 Jiazi Road, Foshan, Guangdong 528308, P.R. China

E-mail: ewv8x7@163.com

Key words: posterior communicating artery, posterior circulation ischemia, phase contrast technique, magnetic resonance angiography
F-PCoA can be analyzed via PC-MRA. The forward posterior shunt flow of F-PCoA can provide references for the clinical auxiliary diagnosis.

\section{Introduction}

The cerebral blood supply comes from the internal carotid artery system (anterior circulation) and vertebrobasilar system (posterior circulation). Not only sex, age, diabetes, hypertension, hyperlipidemia and other recognized factors affecting morbidity but also posterior communicating artery (PCoA) is an important collateral vessel linking anterior and posterior circulation blood, which may be related to posterior circulation ischemia (PCI) (1-5). Functional posterior communicating artery (F-PCoA) may be divided into the following 3 types: F-PCoA was consistent with anatomical posterior communicating artery (A-PCoA), F-PCoA was inconsistent with A-PCoA, and mixed type, which is different from F-PCoA and A-PCoA. For example, A-PCoA is only a potential component, namely, the same blood pressure at both ends, no flow in blood vessels, or too small vascular diameter, and very low shunt flow volume, indicating the presence of A-PCoA but absence of F-PCoA. The hemodynamic information of the F-PCoA, such as blood flow direction, velocity and volume, can be quantitatively analyzed via phase contrast magnetic resonance angiography (PC-MRA) without using the contrast agent, which is of important value in evaluating the collateral circulation function of PCoA (6). PC-MRA is a non-radioactive detection method for cerebral blood flow, which can detect the acute ischemic lesions and provide both morphological and hemodynamic information simultaneously. The velocity-time curve of target vessels can be obtained, and such hemodynamic parameters as the mean flow velocity and mean flow volume can be calculated using the flow analysis software, which is of important value in evaluating the collateral compensatory F-PCoA (7).

In this study, the hemodynamic features of PCoA in PCI patients were studied via PC-MRA to provide clinical references. This study is helpful to identify subjects with clinically suspected PCI but negative results in routine MR plain scan, or subjects without clinical symptoms but with decreased posterior circulation blood flow volume. 
Table I. PC-MRAs of F-PCoA.

\begin{tabular}{lccc}
\hline Factors & Left F-PCoA & Right F-PCoA & Total \\
\hline Blood flow direction & & & 7 \\
Bidirectional blood flow (n) & 7 & 19 & 35 \\
Backwards blood flow (n) & 16 & 0 & 3 \\
Forwards blood flow (n) & 3 & $(1.92,72.0)$ & $(0.24,81.6)$ \\
Mean flow (ml/min) & $(0.24,81.6)$ & 7.50 & 20.31 \\
(Min, Max) & 10.44 & $(-5.98,19.10)$ & $(-9.67,19.10)$ \\
Median & $(-9.67,11.2)$ & 1.27 & 4.01 \\
Mean velocity (cm/sec) & 1.07 & $(0.02,0.21)$ & $(0.02,0.21)$ \\
(Min, Max) & $(0.04,0.14)$ & 0.07 & 0.08 \\
Median & 0.07 & & $(0.11,0.31)$ \\
Cross-sectional area (cm $\left.{ }^{2}\right)$ & & $(0.11,0.31)$ & 0.16 \\
Min, Max) & $(0.11,0.24)$ & 0.16 & \\
Median & 0.16 & & \\
Diameter (cm) & & & \\
(Min, Max) & & & \\
Median & & & \\
\hline
\end{tabular}

F-PCoA, functional posterior communicating artery.

\section{Patients and methods}

Enrolment of patients. Patients receiving magnetic resonance imaging (MRI) plain scan and PC-MRA in Shunde Hospital, Southern Medical University (The First People's Hospital of Shunde Foshan) (Foshan, China) from April 2015 to March 2017 were collected. Exclusion criteria for the study were: i) those with abnormal collateral circulation or vascular malformation in the brain, ii) those with hemorrhagic cerebrovascular disease, or iii) with brain tumor, brain trauma or a history of brain surgery. Finally, 51 subjects were enrolled. The study was approved by the Ethics Committee of Shunde Hospital, Southern Medical University (The First People's Hospital of Shunde Foshan). Signed informed consents were obtained from the patients or the guardians.

The functional PCoA (F-PCoA) was defined as the complete arterial blood vessel with a diameter $>1 \mathrm{~mm}$ and both ends connecting to the cerebral anterior and posterior circulation vessels, whose flow volume and velocity could be measured via PC-MRA. The anatomical PCoA (A-PCoA) was defined as the artery between the anterior circulation vessel (internal carotid artery, middle cerebral artery or anterior cerebral artery) and the $\mathrm{P} 1$ segment of the ipsilateral posterior cerebral artery, including dysplastic vessels.

Among those with F-PCoA, 27 subjects diagnosed with PCI based on the 'Chinese Guidelines for Diagnosis and Treatment of Acute Ischemic Stroke in 2014' (8) as the golden standard were included into research group, including 16 males and 11 females at an average age of $65.56 \pm 13.47$ years, and their clinical data were reviewed in detail one by one. Eleven non-PCI subjects clinically diagnosed according to the same golden standard at the similar age and with similar blood pressure, blood glucose and blood lipid to those in research group were included into control group 1, including 4 males and 7 females at an average age of $59.45 \pm 7.13$ years. The remaining 13 non-PCI subjects without F-PCoA were included as control group 2, including 5 males and 8 females at an average age of 64.92 \pm 7.35 years. Control groups 1 and 2 were combined as control group $(n=24)$.

Data collection. Philips Intera Achieva 1.5T MRI, Fast CINE PC-MRA sequence and pulse peripheral gating were used. On the three-dimensional time-of-flight MRA maximum signal projection reconstructed images, combined with twodimensional tomographic source images, the basal artery (BA), left and right vertebral arteries, internal carotid artery and F-PCoA were located and detected. Fast CINE PC-MRA sequence parameters are as follows: the shortest TR and TE were selected automatically, FOV (mm): 100x100, NSA=2, and layer thickness: $2 \mathrm{~mm}$. The target vessel, perpendicular to the long axis of the measuring segment of target vessel, was placed in the central position of the layer, and the appropriate heart phase value was adjusted according to the finger pulse. The velocity encoding (Venc) was set as $100 \mathrm{~cm} / \mathrm{sec}$, and the total sequence running time was approximately $3 \mathrm{~min}$ and $30 \mathrm{sec}$.

Mean flow volume, mean flow velocity, minimum flow volume, maximum flow volume, minimum flow velocity and maximum flow velocity of target vessels were recorded one by one, the peak height of flow volume (maximum flow volume - minimum flow volume) and flow velocity (maximum flow velocity - minimum flow velocity) were calculated, and the blood flow direction was determined according to the plus-minus sign of flow velocity. The relationship between F-PCoA and A-PCoA was recorded. 
Table II. Statistical analysis of 24 cases without PCI.

\begin{tabular}{|c|c|c|c|c|}
\hline Characteristics & $\begin{array}{l}\text { F-PCoA } \\
(n=11)\end{array}$ & $\begin{array}{l}\text { Non-F-PCoA } \\
\quad(n=13)\end{array}$ & \multicolumn{2}{|c|}{ Statistical analysis } \\
\hline Sex (male/female) & $4 / 7$ & $5 / 8$ & $\chi^{2}=0.011$ & $\mathrm{P}=0.916$ \\
\hline Age (years) & $59.45 \pm 7.13$ & $64.92 \pm 7.35$ & $\mathrm{t}=1.840$ & $\mathrm{P}=0.079$ \\
\hline \multicolumn{5}{|l|}{ BA } \\
\hline Cross-sectional area $\left(\mathrm{mm}^{2}\right)$ & $12.95 \pm 2.98$ & $19.11 \pm 6.85$ & $t=2.762$ & $\mathrm{P}=0.011^{\mathrm{a}}$ \\
\hline Diameter $(\mathrm{mm})$ & $2.86 \pm 0.66$ & $3.49 \pm 0.42$ & $\mathrm{t}=2.846$ & $\mathrm{P}=0.009^{\mathrm{a}}$ \\
\hline Mean flow volume (ml/min) & $144.16 \pm 44.01$ & $165.18 \pm 24.11$ & $\mathrm{t}=1.483$ & $P=0.152$ \\
\hline Mean velocity $(\mathrm{cm} / \mathrm{sec})$ & $19.30 \pm 6.72$ & $15.25 \pm 4.67$ & $\mathrm{t}=2.099$ & $\mathrm{P}=0.147$ \\
\hline Minimum flow volume (ml/min) & $81.98 \pm 40.73$ & $87.97 \pm 18.73$ & $\mathrm{t}=13.538$ & $P=0.661$ \\
\hline Maximum flow volume (ml/min) & $214.58 \pm 56.98$ & $271.80 \pm 47.10$ & $\mathrm{t}=2.695$ & $\mathrm{P}=0.013^{\mathrm{a}}$ \\
\hline Peak height of flow volume ( $\mathrm{ml} / \mathrm{min})$ & $132.60 \pm 31.00$ & $183.83 \pm 42.65$ & $\mathrm{t}=3.308$ & $\mathrm{P}=0.003^{\mathrm{a}}$ \\
\hline Minimum velocity $(\mathrm{cm} / \mathrm{sec})$ & $30.91 \pm 9.46$ & $25.12 \pm 7.17$ & $\mathrm{t}=-1.706$ & $\mathrm{P}=0.102$ \\
\hline Maximum velocity (cm/sec) & $71.80 \pm 15.76$ & $59.62 \pm 11.49$ & $\mathrm{t}=-2.186$ & $\mathrm{P}=0.040^{\mathrm{a}}$ \\
\hline Peak height of velocity $(\mathrm{cm} / \mathrm{sec})$ & $40.89 \pm 9.36$ & $34.51 \pm 7.33$ & $\mathrm{t}=-1.875$ & $\mathrm{P}=0.074$ \\
\hline Flow volume of bilateral internal carotid arteries (ml/min) & $520.68 \pm 89.00$ & $461.43 \pm 64.14$ & $\mathrm{t}=-1.858$ & $\mathrm{P}=0.077$ \\
\hline Flow volume of bilateral vertebral arteries (ml/min) & $211.16 \pm 72.94$ & $221.27 \pm 46.60$ & $\mathrm{t}=0.345$ & $\mathrm{P}=0.735$ \\
\hline
\end{tabular}

${ }^{\mathrm{a}} \mathrm{P}<0.05$. PCI, posterior circulation ischemia; BA, basal artery; F-PCoA, functional posterior communicating artery.

Statistical analysis. Statistical Product and Service Solutions (SPSS) 13.0 software was used. In statistical inference, t-test was used for measurement data in line with the normal distribution and homogeneity of variance, and rank sum test was used otherwise. Chi-square test was used for constituent ratio data. Two-sided tests were used. $\mathrm{P}<0.05$ was considered to indicate a statistically significant difference.

\section{Results}

$P C$-MRAs of F-PCoA. Among subjects receiving PC-MRA, there were 38 subjects with a total of 52 F-PCoAs, and they were divided into the following 3 types: i) F-PCoA was consistent with A-PCoA, accounting for $84.21 \%$ (32/38), ii) F-PCoA was inconsistent with A-PCoA, accounting for $13.16 \%(5 / 38)$, and iii) mixed type, accounting for $2.63 \%$ (1/38). The blood flow curve of F-PCoA displayed sawtooth-like changes, and there could be either unidirectional blood flow or bidirectional blood flow in one cardiac cycle. Among the 16 subjects with bilateral F-PCoA, the bilateral blood flow direction was the same in 10 cases $(62.50 \%)$ (Table I).

Parameters. There were no significant differences in sex composition, age distribution, total flow volume of bilateral internal carotid arteries, total flow volume of bilateral vertebral arteries, and mean flow volume, mean flow velocity, minimum flow volume, minimum flow velocity and peak height of flow velocity of BA between control groups 1 and 2 . However, the cross-sectional area, diameter, maximum flow volume, peak height of flow volume and maximum flow velocity of BA had significant differences (Table II).

There were no significant differences in sex composition, age distribution, total flow volume of bilateral internal carotid

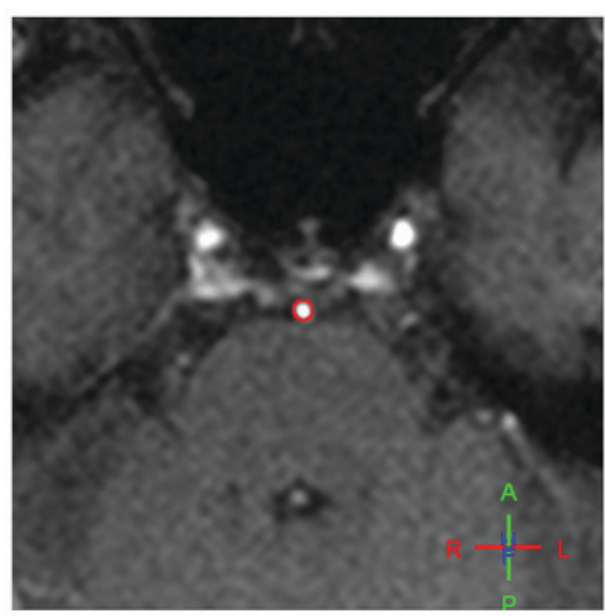

Figure 1. ROI of basal artery.

arteries, total flow volume of bilateral vertebral arteries and cross-sectional area of BA between research group and control group. However, the mean flow volume, mean flow velocity, minimum flow volume, maximum flow volume and minimum flow velocity of BA, and forward posterior shunt flow volume of F-PCoA had significant differences (Table III).

Cases. One case in the research group involved a 54-year-old male patient suffering from repeated dizziness for 1 year, which was aggravated for 10 days. MRI manifestations were softening lesion in the left cerebellum, multiple stenosis in the bilateral posterior cerebral arteries, and segmental stenosis in the left vertebral artery. PC-MRA manifestations 
Table III. Statistical analysis of 51 cases.

\begin{tabular}{lcccc}
\hline Characteristics & $\begin{array}{c}\text { Experimental } \\
\text { group }(\mathrm{n}=27)\end{array}$ & $\begin{array}{c}\text { Control group } \\
(\mathrm{n}=24)\end{array}$ & Statistical analysis \\
\hline Sex (male/female) & $16 / 11$ & $9 / 15$ & $\chi^{2}=2.407$ & $\mathrm{P}=0.121$ \\
Age (years) & $65.56 \pm 13.47$ & $62.42 \pm 7.62$ & $\mathrm{t}=-1.038$ & $\mathrm{P}=0.305$ \\
BA & & & & \\
Cross-sectional area $\left(\mathrm{mm}^{2}\right)$ & $20.20 \pm 9.46$ & $16.28 \pm 6.18$ & $\mathrm{t}=-1.768$ & $\mathrm{P}=0.084$ \\
Mean flow volume $(\mathrm{ml} / \mathrm{min})$ & $119.38 \pm 33.78$ & $155.55 \pm 35.49$ & $\mathrm{t}=3.726$ & $\mathrm{P}=0.001^{\mathrm{a}}$ \\
Mean velocity $(\mathrm{cm} / \mathrm{sec})$ & $11.69 \pm 5.33$ & $17.10 \pm 5.94$ & $\mathrm{t}=3.432$ & $\mathrm{P}=0.001^{\mathrm{a}}$ \\
Minimum flow volume $(\mathrm{ml} / \mathrm{min})$ & $54.33 \pm 33.21$ & $85.23 \pm 30.23$ & $\mathrm{t}=3.458$ & $\mathrm{P}=0.001^{\mathrm{a}}$ \\
Maximum flow volume $(\mathrm{ml} / \mathrm{min})$ & $208.31 \pm 69.67$ & $245.58 \pm 58.46$ & $\mathrm{t}=2.055$ & $\mathrm{P}=0.045^{\mathrm{a}}$ \\
Peak height of flow volume $(\mathrm{ml} / \mathrm{min})$ & $153.98 \pm 73.52$ & $160.35 \pm 45.24$ & $\mathrm{t}=1.178$ & $\mathrm{P}=0.278$ \\
Minimum velocity $(\mathrm{cm} / \mathrm{sec})$ & $21.06 \pm 7.47$ & $27.77 \pm 8.63$ & $\mathrm{t}=2.976$ & $\mathrm{P}=0.005^{\mathrm{a}}$ \\
Maximum velocity $(\mathrm{cm} / \mathrm{sec})$ & $59.61 \pm 23.62$ & $65.20 \pm 14.67$ & $\mathrm{t}=1.001$ & $\mathrm{P}=0.322$ \\
Peak height of velocity $(\mathrm{cm} / \mathrm{sec})$ & $38.54 \pm 20.88$ & $37.43 \pm 8.75$ & $\mathrm{t}=0.556$ & $\mathrm{P}=0.456$ \\
F-PCoA & & & & \\
Backward shunt flow $(\mathrm{ml} / \mathrm{min})$ & $27.15 \pm 35.33$ & $15.12 \pm 29.05$ & $\mathrm{t}=6.216$ & $\mathrm{P}=0.013^{\mathrm{a}}$ \\
Flow volume of bilateral internal carotid arteries $(\mathrm{ml} / \mathrm{min})$ & $451.24 \pm 124.41$ & $487.19 \pm 79.92$ & $\mathrm{t}=1.191$ & $\mathrm{P}=0.239$ \\
Flow volume of bilateral vertebral arteries $(\mathrm{ml} / \mathrm{min})$ & $191.28 \pm 48.35$ & $216.51 \pm 58.66$ & $\mathrm{t}=1.392$ & $\mathrm{P}=0.173$ \\
\hline
\end{tabular}

${ }^{\mathrm{a}} \mathrm{P}<0.05$. BA, basal artery; F-PCoA, functional posterior communicating artery.

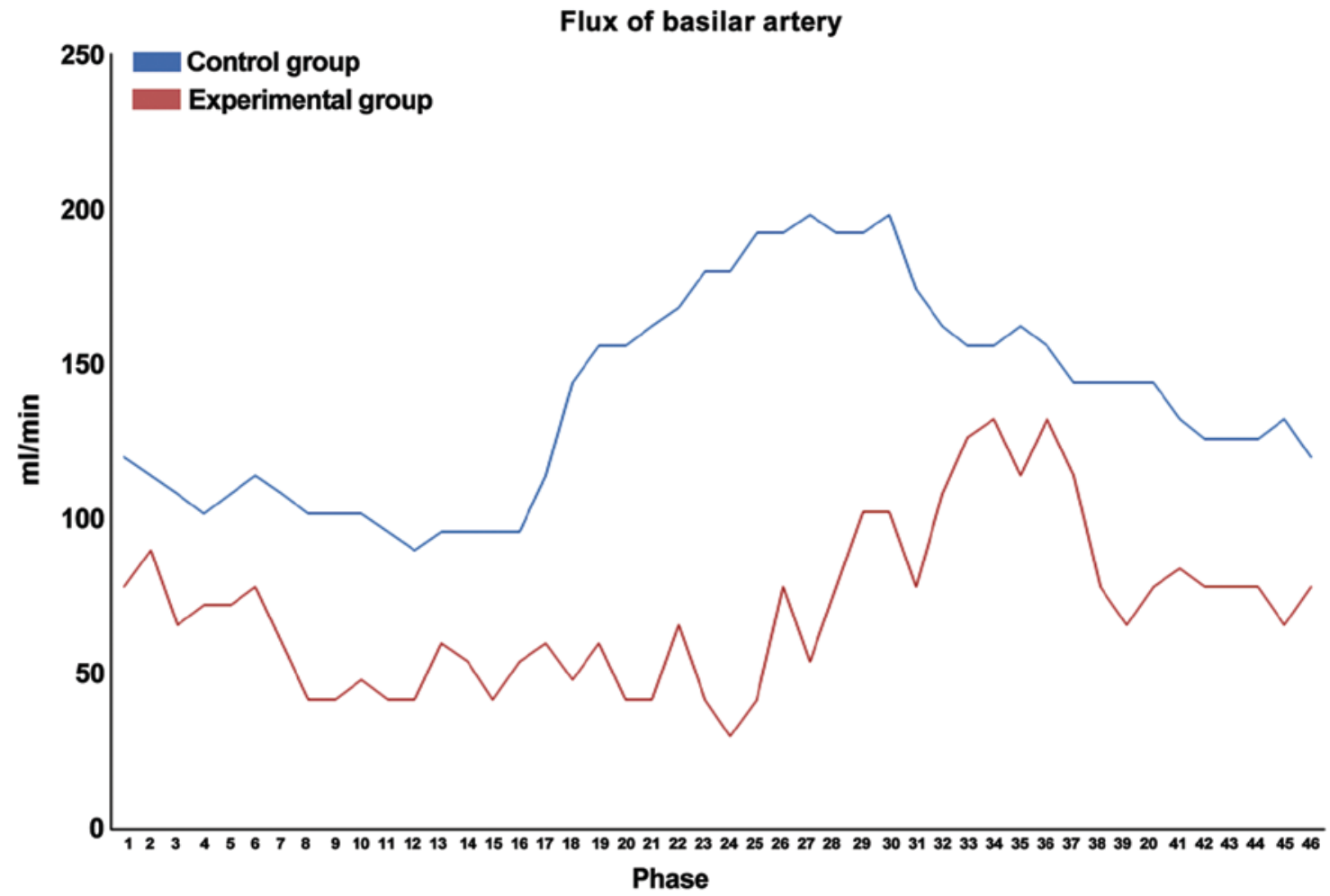

Figure 2. Flow of basal artery.

showed moderate decline in the total blood flow volume in the posterior circulation, slight decline in the mean flow velocity of BA, and compensatory changes in the posterior circulation blood-supply area in the bilateral PCoA.
One case in the control group was a 77-year-old female patient suffering from repeated dizziness for 6 months, which was aggravated for 2 days. No abnormalities were identified in MRI and PC-MRA (Figs. 1-5). 


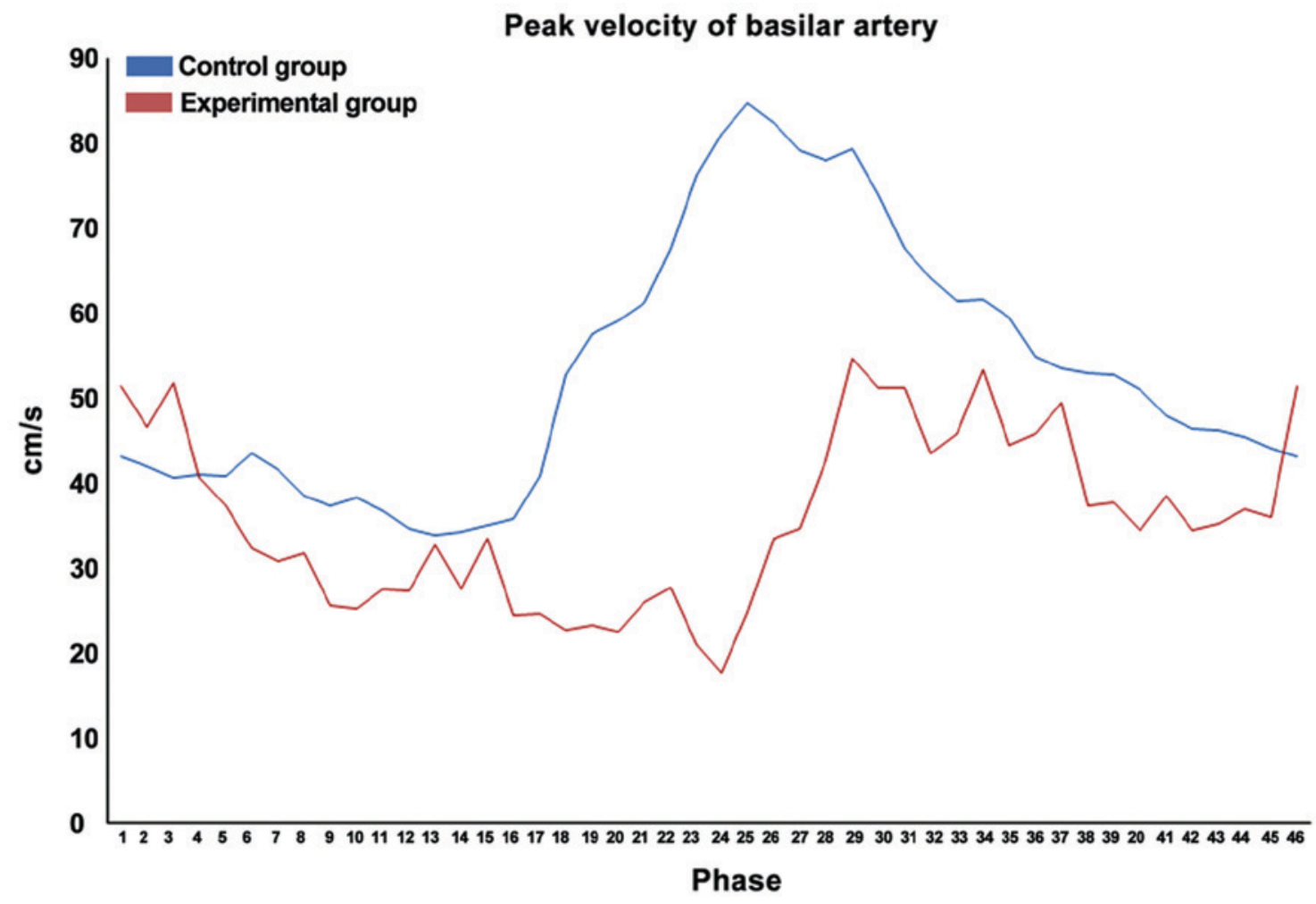

Figure 3. Peak velocity of basal artery.

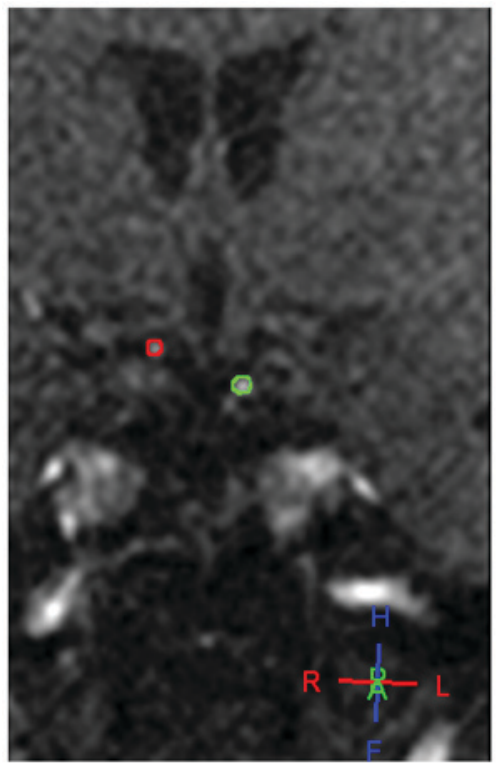

Figure 4. ROI of F-PCoA.

\section{Discussion}

Research methods of cerebral hemodynamics (9-13) include positron mission tomography/computer tomography (PET/CT), transcranial Doppler ultrasonography (TCD), CT/MRI perfusion and PC-MRA. PET/CT is the golden standard for clinical detection of cerebral blood flow volume, which can provide morphological and metabolic parameters. However, the examination cost is high, only a few large hospitals are qualified for the examination, and a control study for healthy individuals does not conform to medical ethics. The single target vessel can be detected via TCD, but PCoA has a small diameter, tortuous walking and complex variation, so it is difficult to locate it. Currently, PCoA has not been applied in the clinical detection yet. The whole or local macroscopic cerebral blood flow information can be obtained via CT or MRI perfusion, but it is more difficult to study the responsible vessels in a targeted manner. PC-MRA is a non-radioactive detection method for cerebral blood flow, which can detect the acute ischemic lesions and provide both morphological and hemodynamic information simultaneously. The velocity-time curve of target vessels can be obtained, and such hemodynamic parameters as the mean flow velocity and mean flow volume can be calculated using flow analysis software, which is of important value in evaluating the collateral compensatory function of PCoA (7). In addition, digital subtraction angiography (DSA) is characterized by high spatial resolution, temporal resolution, as well as sensitivity and specificity, which is the gold standard for the vascular morphological examination. However, DSA cannot be used in the examination for normal control subjects, because it can cause large trauma, and the contrast agent can only be injected in sequence to display the left and right PCoA one by one, with poor overall view.

F-PCoA is an important collateral blood vessel linking the anterior-posterior circulation blood flow. Under different conditions, there may be forward posterior, backward anterior or bidirectional shunt in F-PCoA, and the influence of F-PCoA cannot be ignored. Table II indicates that in the presence of F-PCoA, the diameter and cross-sectional area of BA are small, and the maximum flow volume and peak height of flow volume are reduced, but the maximum flow velocity is increased, which can be regarded as a kind of adaptive 


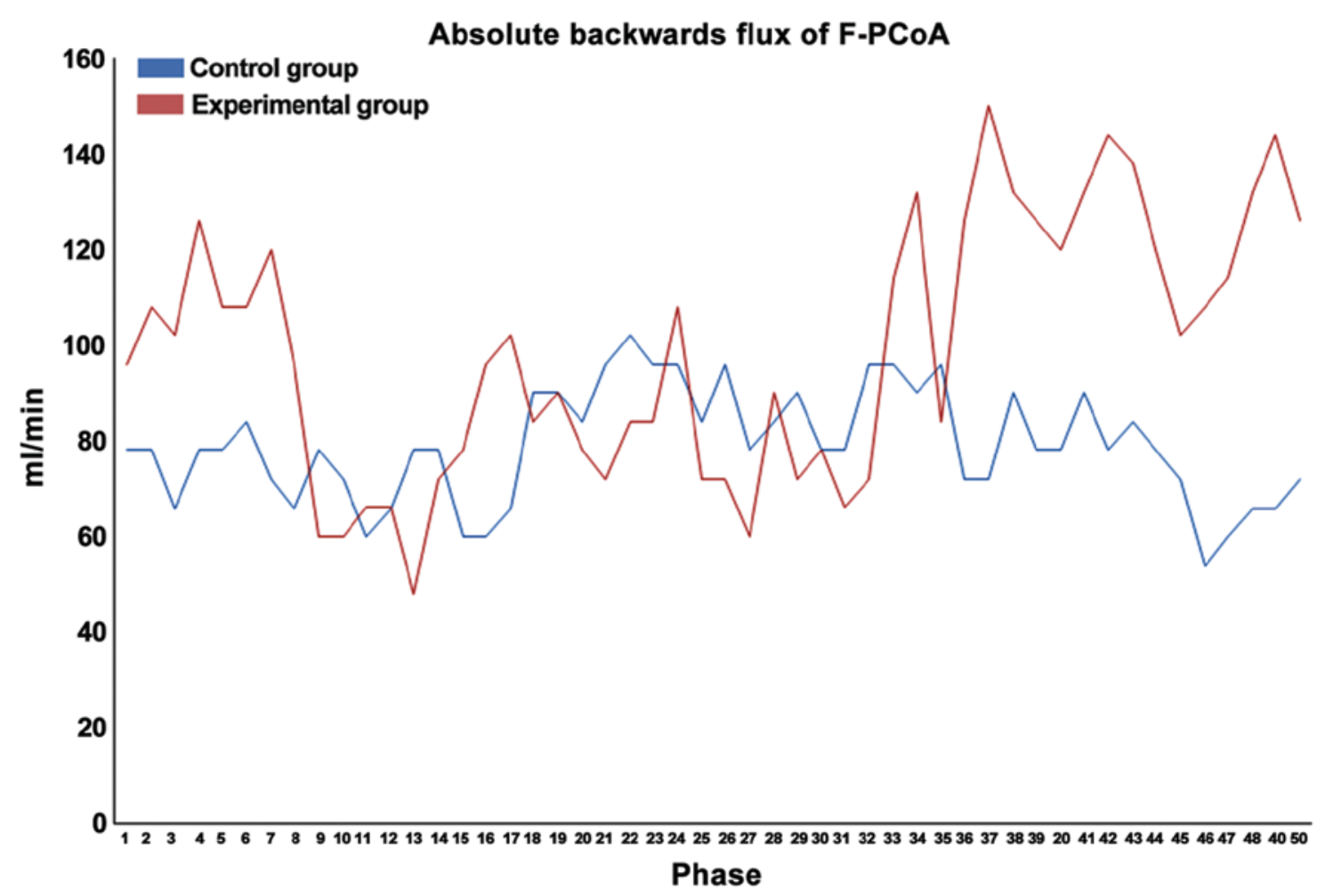

Figure 5. Absolute backward flow of F-PCoA.

change that the internal carotid artery blood flow reaches the blood-supply area of posterior circulation through forward posterior shunt of F-PCoA to maintain the stability of the total blood flow volume in the posterior circulation. There is no necessary correlation between the smaller diameter of BA and the occurrence of PCI. Whether there are shunt and compensation between the anterior and posterior circulations is important clinical diagnostic information. The following 4 conditions can indicate no shunt between the anterior and posterior circulation based on the PC-MRA results in this study: i) there is no PCoA, ii) PCoA is only a potential, namely the same blood pressure at both ends, no flow in blood vessels, or too small vascular diameter, and very low shunt flow volume, indicating the presence of A-PCoA but absence of F-PCoA, iii) the blood pressure at both ends of F-PCoA is similar, and the bidirectional blood flow reaches the dynamic balance in one cardiac cycle, and iv) the flow volume of bilateral F-PCoA is the same but the flow direction is the opposite. The above results suggest that the differences and relationship between F-PCoA and A-PCoA should be paid attention to, and the theory in previous studies $(1,6,9)$ that there is shunt in the presence of PCoA and PCoA is a protective blood flow pathway for BA stenosis should be further improved.

Results revealed that in the presence of F-PCoA, there were no significant differences in the total blood flow of bilateral internal carotid arteries and vertebral arteries and the diameter of BA between PCI and non-PCI patients, but the mean flow volume, mean flow velocity, minimum flow volume, maximum flow volume and minimum flow velocity of BA in PCI patients were all decreased. At the same time, the forward posterior shunt flow volume of F-PCoA was significantly increased (Table III). Results also showed that there was a more obvious main peak of F-PCoA in the research group than that in the control group, indicating that the forward and backward shunt flow volume in the systolic phase is increased in one cardiac cycle, while that in the diastolic phase is similar to that in control group. The above results suggest that the blood flow in F-PCoA can compensate for the decreased blood flow of BA to a certain degree and play a protective role in PCI, and such effects are more significant in the systolic phase.

If the blood flow of BA is reduced, forward posterior shunt is detected in F-PCoA, and there are clinical manifestations of PCI, but no positive infarct is detected via conventional MRI, it may be in the decompensatory stage of posterior circulation blood flow, and active intervention should be made for risk factors of PCI. Kaspera et al (14) showed that in the bilateral internal carotid arterial occlusion, the anterior circulation ischemia can be partially compensated by posterior circulation blood flow through backward anterior shunt of PCoA. According to further research $(1,6,15)$, such a shunt may increase the risk of posterior circulation ischemia. In this study, the shunt from backward to forward was detected in some F-PCoAs, the blood flow of internal carotid artery was reduced, there was no significant or slight increase in the blood flow of BA, there were clinical manifestations of posterior circulation ischemia, or posterior circulation infarct was detected via MRI, thereby providing additional evidence for the above theory.

This study is useful in the identification of subjects with clinically suspected PCI but negative results in routine MR plain scan, or subjects without clinical symptoms but with decreased posterior circulation blood flow volume, the former of which is the incomplete compensation of blood flow and the latter of which is the complete compensation of blood flow. 
In either case, however, if subjects are not closely followed up and risk factors are not controlled in a timely manner, PCI may progress to irreversible cerebral infarction. This study also had some limitations: i) the sample size was small, and the supporting evidence for statistical analysis was insufficient. ii) There was a lack of reliable and recognized gold standard for the PC-MRA results, and it was only a single-center study; thus, results remain to be further verified.

In conclusion, this study indicates that hemodynamic characteristics of F-PCoA can be analyzed using the mature technique of PC-MRA. The forward posterior shunt flow volume of F-PCoA can provide references for the clinical auxiliary diagnosis.

\section{Acknowledgements}

Not applicable.

\section{Funding}

This study was supported by the Medical Science and Technology Breakthrough project of Foshan Science and Technology Bureau (nos. 2015AB002553, 2014AB002553).

\section{Availability of data and materials}

The datasets used and/or analyzed during the present study are available from the corresponding author on reasonable request.

\section{Authors' contributions}

WZ drafted the manuscript. WZ, ML and JL contributed to collecting and interpreting the data. FC, QH and SY were responsible for statistical analysis. All authors read and approved the final manuscript.

\section{Ethics approval and consent to participate}

The study was approved by the Ethics Committee of Shunde Hospital, Southern Medical University (The First People's Hospital of Shunde Foshan) (Foshan, China). Signed informed consents were obtained from the patients or the guardians.

\section{Patient consent for publication}

Not applicable.

\section{Competing interests}

The authors declare that they have no competing interests.

\section{References}

1. Hu XY, Li ZX, Liu HQ, Zhang M, Wei ML, Fang S, Chen W, Pan H, Huang JX, Zhu YM, et al: Relationship between vertebral artery hypoplasia and posterior circulation stroke in Chinese patients. Neuroradiology 55: 291-295, 2013.

2. Iqbal S: A comprehensive study of the anatomical variations of the circle of Willis in adult human brains. J Clin Diagn Res 7: 2423-2427, 2013.

3. Nouh A, Remke J and Ruland S: Ischemic posterior circulation stroke: A review of anatomy, clinical presentations, diagnosis, and current management. Front Neurol 5: 30, 2014.

4. Zhou W, Xu YK, Lu MR, Liang YJ, Chen F, Hu QG and Liu J: MRA Study on correlation of posterior communicating artery variation and posterior circulation infarction. J Sun Yatsen Univ (Med Sci) 37: 277-282, 2016 (In Chinese).

5. Zhou W and Xu YK: MRA evaluation of variation of posterior communicating artery and its correlation with posterior circulation infarction. Int J Med Radiol 39: 373-377, 2016 (In Chinese).

6. Goerlitz J, Wenz H, Al-Zghloul M, Kerl HU, Groden C and Förster A: Anatomical variations in the posterior circle of Willis and vascular pathologies in isolated unilateral thalamic infarction. J Neuroimaging 25: 983-988, 2015.

7. Orosz L, Hoksbergen AW, Molnár C, Siró P, Cassot F, MarcVergnes JP and Fülesdi B: Clinical applicability of a mathematical model in assessing the functional ability of the communicating arteries of the circle of Willis. J Neurol Sci 287: 94-99, 2009.

8. Division of Cerebrovascular Disease and Branch of Neurology: Chinese Medical Association: Chinese guidance for diagnosis and treatment of acute ischemic stroke in 2014. Chin J Neurol 48: 246-257, 2015.

9. Leung J, Behpour A, Sokol N, Mohanta A and Kassner A: Assessment of intracranial blood flow velocities using a computer controlled vasoactive stimulus: A comparison between phase contrast magnetic resonance angiography and transcranial Doppler ultrasonography. J Magn Reson Imaging 38: 733-738, 2013.

10. Kim HJ, Kim TW, Ryu SY, Yang PS, Kwon MJ, Kim JC, Lee YS, Lee HJ, Yang JH, Kim JK, et al: Acetazolamide-challenged perfusion magnetic resonance imaging for assessment of cerebrovascular reserve capacity in patients with symptomatic middle cerebral artery stenosis: Comparison with technetium99m-hexamethylpropyleneamine oxime single-photon emission computed tomography. Clin Imaging 35: 413-420, 2011.

11. Rijbroek A, Wisselink W, Vriens EM, Barkhof F, Lammertsma AA and Rauwerda JA: Asymptomatic carotid artery stenosis: Past, present and future. How to improve patient selection? Eur Neurol 56: 139-154, 2006.

12. Ursino M, Lodi CA and Russo G: Cerebral hemodynamic response to $\mathrm{CO}(2)$ tests in patients with internal carotid artery occlusion: Modeling study and in vivo validation. J Vasc Res 37: 123-133, 2000.

13. Purkayastha S and Sorond F: Transcranial Doppler ultrasound: Technique and application. Semin Neurol 32: 411-420, 2012.

14. Kaspera W, Ładziński P, Larysz P, Majchrzak H, Hebda A, Kopera M, Tomalski W and Ślaska A: Transcranial color-coded Doppler assessment of cerebral arteriovenous malformation hemodynamics in patients treated surgically or with staged embolization. Clin Neurol Neurosurg 116: 46-53, 2014.

15. Asif A, Leon C, Merrill D, Bhimani B, Ellis R, Ladino M and Gadalean FN: Arterial steal syndrome: A modest proposal for an old paradigm. Am J Kidney Dis 48: 88-97, 2006. International (CC BY-NC-ND 4.0) License. 